\title{
CAMP/CREB-regulated LINC00473 marks LKB1- inactivated lung cancer and mediates tumor growth
}

\author{
Zirong Chen, ${ }^{1,2}$ Jian-Liang Li, ${ }^{3}$ Shuibin Lin, ${ }^{1,2}$ Chunxia Cao, ${ }^{2,4}$ Nicholas T. Gimbrone, ${ }^{5}$ Rongqiang Yang, ${ }^{1,2}$ Dongtao A. Fu, ${ }^{6}$ \\ Miranda B. Carper, ${ }^{7}$ Eric B. Haura, ${ }^{8}$ Matthew B. Schabath, ${ }^{9}$ Jianrong Lu, ${ }^{2,10}$ Antonio L. Amelio, ${ }^{7}$ W. Douglas Cress, ${ }^{5}$ \\ Frederic J. Kaye, ${ }^{2,4}$ and Lizi $\mathrm{Wu}^{1,2}$ \\ 'Department of Molecular Genetics and Microbiology and 2UF Health Cancer Center, College of Medicine, University of Florida, Gainesville, Florida, USA. ${ }^{3}$ Sanford Burnham Prebys Medical Discovery Institute \\ at Lake Nona, Orlando, Florida, USA. ${ }^{4}$ Department of Medicine, College of Medicine, University of Florida, Gainesville, Florida, USA. ${ }^{5}$ Department of Molecular Oncology, Moffitt Cancer Center, Tampa, \\ Florida, USA. ${ }^{6}$ Department of Pathology, Immunology and Laboratory Medicine, College of Medicine, University of Florida, Gainesville, Florida, USA. Lineberger Comprehensive Cancer Center, University \\ of North Carolina at Chapel Hill, Chapel Hill, North Carolina, USA. ${ }^{8}$ Department of Thoracic Oncology and ${ }^{9}$ Department of Cancer Epidemiology, Moffitt Cancer Center, Tampa, Florida, USA. ${ }^{10}$ Department of \\ Biochemistry and Molecular Biology, College of Medicine, University of Florida, Gainesville, Florida, USA.
}

\begin{abstract}
The LKB1 tumor suppressor gene is frequently mutated and inactivated in non-small cell lung cancer (NSCLC). Loss of LKB1 promotes cancer progression and influences therapeutic responses in preclinical studies; however, specific targeted therapies for lung cancer with LKB1 inactivation are currently unavailable. Here, we have identified a long noncoding RNA (IncRNA) signature that is associated with the loss of LKB1 function. We discovered that LINCO0473 is consistently the most highly induced gene in LKB1-inactivated human primary NSCLC samples and derived cell lines. Elevated LINC00473 expression correlated with poor prognosis, and sustained LINC00473 expression was required for the growth and survival of LKB1inactivated NSCLC cells. Mechanistically, LINC00473 was induced by LKB1 inactivation and subsequent cyclic AMP-responsive element-binding protein (CREB)/CREB-regulated transcription coactivator (CRTC) activation. We determined that LINCO0473 is a nuclear IncRNA and interacts with NONO, a component of the CAMP signaling pathway, thereby facilitating CRTC/ CREB-mediated transcription. Collectively, our study demonstrates that LINC00473 expression potentially serves as a robust biomarker for tumor LKB1 functional status that can be integrated into clinical trials for patient selection and treatment evaluation, and implicates LINC00473 as a therapeutic target for LKB1-inactivated NSCLC.
\end{abstract}

\section{Introduction}

Lung cancer is the leading cause of cancer-related deaths (1). Approximately $80 \%$ of lung cancer cases are non-small cell lung carcinoma (NSCLC). Conventional cytotoxic therapeutics show limited effectiveness; but recent targeted therapies utilizing inhibitors targeting specific driver gene activating mutations, such as $E G F R$ and $A L K$, have demonstrated promising improved clinical outcomes (2-4). However, effective targeted therapies for other common somatic mutations such as LKB1 (STK11) in lung cancer remain an unmet medical need.

The $L K B 1$ gene encodes a serine-threonine kinase that plays critical roles in cell growth, polarity, and metabolism $(5,6) . L K B 1$ is a tumor suppressor gene whose mutations cause Peutz-Jeghers syndrome, which is associated with increased cancer risk $(7,8)$. LKB1 is a target for mutational inactivation in sporadic cancers, especially NSCLC, where it is mutated in approximately $20 \%-30 \%$

Note regarding evaluation of this manuscript: Manuscripts authored by scientists associated with Duke University, The University of North Carolina at Chapel Hill, Duke-NUS, and the Sanford-Burnham Medical Research Institute are handled not by members of the editorial board but rather by the science editors, who consult with selected external editors and reviewers.

Conflict of interest: The authors have declared that no conflict of interest exists. Submitted: October 27, 2015; Accepted: March 10, 2016.

Reference information: / Clin Invest. 2016;126(6):2267-2279. doi:10.1172/JCI85250. of cases, making it the third most common site of genetic alterations after TP53 and KRAS (9-12). LKB1 mutations have been linked with lung cancer progression and differential treatment responses. $L K B 1$ loss was found to promote lung cancer metastasis in a KRAS mouse model of NSCLC (13). Importantly, LKB1 inactivation influenced the responsiveness of cancer cells to treatments in vitro and in vivo. For instance, LKB1 loss sensitized NSCLC responses to the metabolism drug phenformin and a COX2 inhibitor $(14,15)$. On the other hand, $L K B 1$ loss impaired the responses of KRAS mutant NSCLC to docetaxel monotherapy; the combination therapy of docetaxel and the MEK inhibitor selumetinib; or dual inhibition of the PI3K and MEK pathways $(16,17)$. Additionally, LKB1 mutations were associated with the modulation of immune microenvironments, thus potentially impacting immunotherapeutic outcomes $(18,19)$. The current data emphasize the need to determine tumor $L K B 1$ status for patient stratification for evaluating therapeutic responses and identifying effective treatments. However, detection of LKB1 functional loss is technically challenging because LKB1 function can be inactivated by multiple mechanisms, such as somatic gene mutations, epigenetic silencing, posttranslational modifications, and alterations in LKB1 pathway components (20-22). Clinical trials for evaluating treatment efficacy on LKB1-inactivated NSCLC are lacking due to the absence of reliable assays for accurate determination of tumor LKB1 status. Therefore, sensitive and specific assays for LKB1 
inactivation as well as effective targeted therapeutics are needed for effective management of LKB1-inactivated NSCLC.

Long noncoding RNAs (lncRNAs) are non-protein-coding transcripts longer than 200 nucleotides and represent a novel class of gene regulators (23). The human genome encodes more than 10,000 lncRNAs, and currently only a handful of lncRNAs have been characterized (24-26). IncRNA expression is frequently deregulated in cancer and shows cell- or tissue-type specificity (27-29), suggesting significant roles for lncRNAs in human cancers. Though lncRNAs remain an understudied class of genes, they have been shown to participate in cancer cell proliferation, survival, migration, and invasion, likely through exertion of multiple regulatory functions at the transcriptional, posttranscriptional, and epigenetic levels $(23,30)$. Importantly, targeting cancer-associated lncRNAs, such as HOTAIR and MALAT1, blocked cancer cell growth and survival in vitro and tumor growth and metastasis in vivo $(31,32)$. Therefore, lncRNAs are critical for cancer development and progression and might serve as novel therapeutic targets. Currently, the molecular mechanisms underlying LKB1 tumor suppression remain incompletely understood $(5,33)$. Whether lncRNAs have a role in mediating the loss of LKB1 tumor suppression in lung cancer has not been studied.

In this study, we performed lncRNA transcriptional profiling to identify lncRNAs that are associated with inactivated LKB1 signaling. We subsequently focused on one novel LKB1regulated lncRNA (LINC00473) because it was consistently the most highly induced target identified with LKB1 loss. The elevated expression of LINC00473 was associated with poor patient prognosis and tightly correlated with LKB1 inactivation status in human lung cancer specimens and derived cell lines. Our expression, functional, and mechanistic data demonstrate that LINC00473 is a potentially robust biomarker for detection of lung cancers with inactivated LKB1, a novel therapeutic target, and a novel gene regulator.

\section{Results}

Genome-wide lncRNA profiling identified LINCO0473 as a top LKB1 signaling-regulated IncRNA in NSCLC cells. The involvement of lncRNAs in altered LKB1 signaling in lung cancer has not to our knowledge been studied. To investigate whether lncRNAs contribute to loss of LKB1 tumor suppression in lung tumorigenesis and maintenance, we performed genome-wide lncRNA transcriptional profiling to identify lncRNAs associated with aberrant LKB1 signaling. We generated three groups of cells by transducing LKB1null A549 NSCLC cells with retroviruses harboring WT LKB1, an LKB1 kinase-dead mutant (K78I), or a vector control. Western blot analysis confirmed expression and kinase activity of LKB1-WT and -K78I proteins in an AMPK activation assay under glucosefree culture conditions (Figure 1A and Supplemental Figure 1; supplemental material available online with this article; doi:10.1172/ JCI85250DS1). We subsequently used Arraystar Human LncRNA Expression Microarrays to profile changes in expression of approximately 30,000 lncRNAs in our three experimental conditions. Using an absolute fold change of at least 2.0 and a $P$ value less than 0.05 , we identified a total of 164 differentially expressed lncRNAs (64 upregulated and 100 downregulated) in A549 cells upon LKB1-WT expression compared with control (Figure 1B and Supplemental Table 1). We also observed 17 upregulated and 49 downregulated lncRNAs associated with LKB1-K78I mutant expression compared with control (Figure 1C and Supplemental Table 2). Comparing expression profiles of LKB1-WT- and LKB1-K78I-expressing cells, we identified 33 upregulated and 83 downregulated lncRNAs, which suggests that their expression is dependent on LKB1 kinase activity (Supplemental Table 3). Furthermore, we profiled and compared lncRNA expression patterns in two groups of cell lines - LKB1-null (A549, H460) and LKB1WT (H322, H3123) - and observed 1,449 upregulated and 918 downregulated lncRNAs in LKB1-null cell lines (Figure 1D and Supplemental Table 4). Finally, by integrating LKB1-regulated lncRNAs and lncRNAs differentially expressed in LKB1-null cell lines (Supplemental Table 1 and Supplemental Table 4), we identified a list of LKB1-regulated lncRNAs (10 upregulated and 1 downregulated) that were differentially expressed between LKB1-null and -WT lung cancer cells (Supplemental Table 5).

Notably, this list contained 3 transcripts for the LINCO0473 gene (also known as C6orf176), which encodes an intergenic lncRNA from the chromosome 6q27 locus. LINC00473 consists of two exons and has two annotated RefSeq transcript isoforms sharing exon 1: transcript variant 1 (tv1; NR_026860, 1822 nt) and tv2 (NR_026861, 1123 nt) (Supplemental Figure 2A). Our 5' and 3 ' rapid amplification of cDNA ends (RACE) assays identified 3 LINC00473 transcript variants, tv1.1, tv2.1, and tv2.2, but not the two annotated transcripts (Supplemental Figure 2, B and C). LINC00473 tv1.1 had a shorter 5' end than the annotated tv1, while tv2.1 and tv2.2 each had $5^{\prime}$ and $3^{\prime}$ ends that were different from the annotated tv2. Coding potential analysis strongly suggested that LINC00473 is a noncoding RNA (Supplemental Figure 2, D and E). Both tv1 and tv2 transcript variants showed significant activation in LKB1-null NSCLC cells (Supplemental Table 5). LINC00473 tv1 was the top differentially expressed lncRNA (>10,000-fold change) when the two LKB1-null (A549 and H460) lines were compared with the two LKB1-expressing cells (H322 and H3123), while LINC00473 tv2 showed about a 40-fold change.

Elevated LINCOO473 expression is tightly correlated with NSCLC LKB1 inactivation status. To validate LKB1-regulated lncRNAs, we utilized a NanoString-based assay that allows direct digital detection of multiple RNA molecules of interest using target-specific, color-coded probe pairs (34). This platform enabled us to simultaneously evaluate multiple LKB1-regulated mRNA and IncRNA candidates (especially LINC00473). Hybridizations were performed on RNA samples from a pair of control and LKB1expressing A549 cells and a panel of NSCLC cell lines (7 LKB1-WT and 7 LKB1-mutant) using a customized codeset. The codeset included several LKB1-regulated lncRNA candidates, known LKB1-regulated protein-coding genes $(35,36)$, as well as three housekeeping genes (GAPDH, GUSB, and TUBB). As shown in the heatmap (Figure 1E, right), LKB1 expression in LKB1-null A549 cells caused significant downregulation of known protein-coding genes (AVPI1, CTH, CPS1, DUSP4, FGA, NR4A2, PDE4B, PDE4D, PTGS2, PTP4A1, SIK1, SLC7A2, SNAI1, TESC, and TFF1), as well as three lncRNAs (LINC00473, AL109792, and BX641110). However, when examined across various cell lines with the confirmed status of cellular LKB1 protein expression (Supplemental 
A

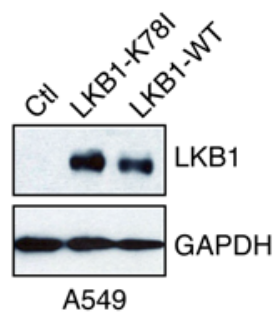

B A549: LKB1-WT vs. ctl

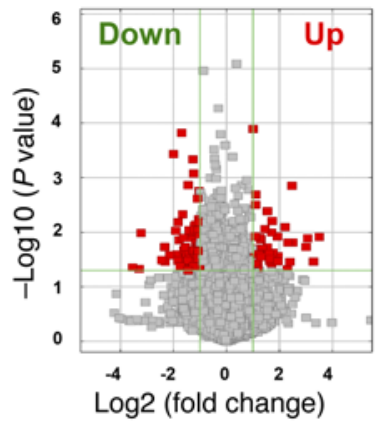

C A549: LKB1-K78I vs. ctl

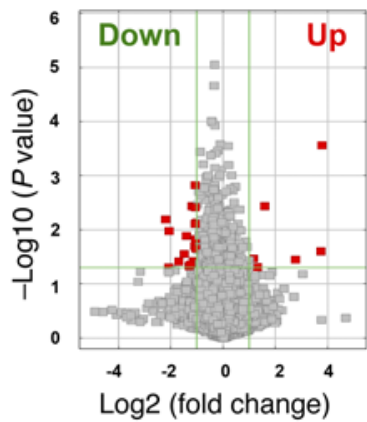

F Lnc473 tv1
D
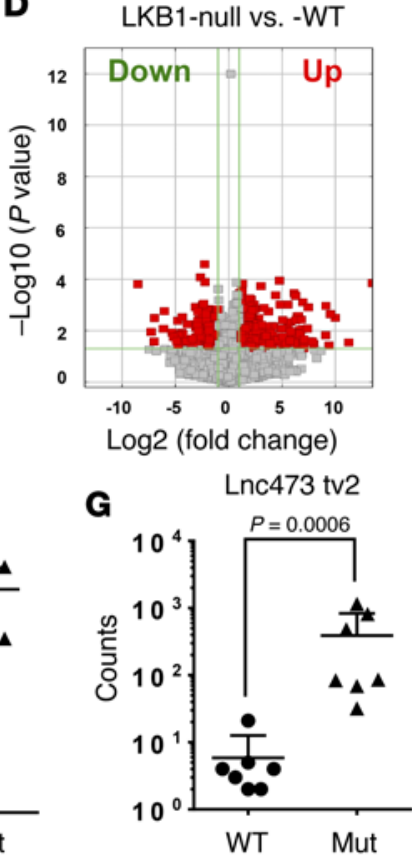

I

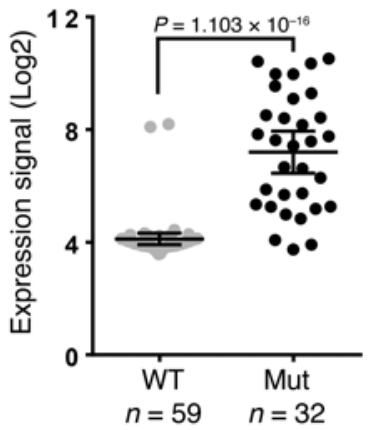

Figure 1. IncRNA profiling revealed that LINC00473 is induced by LKB1 loss in NSCLC cells. (A) Western blot analysis of expression of LKB1-WT or kinasedead K78I mutant in transduced LKB1-null lung adenocarcinoma A549 cells. A549 cells infected with pBabe vector retroviruses were used as controls (Ctl). (B and C) Volcano plots show differentially expressed IncRNAs after expression of LKB1-WT (B) or LKB1-K78I mutant (C) in A549 cells by IncRNA microarray (V3.0) analysis. The cutoff criteria was an absolute fold-change of $\geq 2$ and $P<0.05$. (D) A volcano plot shows differentially expressed IncRNAs in two LKB1-null cell lines (A549 and H460) as compared with two LKB1-WT cell lines (H322 and H3123). (E) A heatmap shows expression levels of several LKB1-regulated protein-coding and noncoding genes measured in NanoString assays. (F and G) Two LINC00473 (Lnc473) transcript variants (tv1: NR_026860 and tv2: NR_026861) show significantly elevated expression in LKB1-mutant (Mut) groups as compared with LKB1-WT groups based on the NanoString assays. (H) CCLE data analysis identified NSCLC cell lines $(n=130)$ with outlier LINC00473 expression levels. (I) Enhanced LINC00473 expression was significantly associated with LKB1 mutations in CCLE NSCLC cell lines. See also Supplemental Figures 1-3 and Supplemental Tables 1-6.

Figure 3A), LINC00473 expression showed the best correlation with LKB1-inactivated status (Figure 1E, left and middle). Other LKB1-regulated genes, including lncRNAs AL109792 and BX641110, were only partially correlated with the LKB1 status, suggesting cell context-dependent gene regulation.

Both the LINC00473 tv1 and tv2 isoforms showed low or absent expression in 7 LKB1-WT NSCLC cell lines but significantly enhanced expression in 7 LKB1-mutant cell lines (Figure 1, F and G). LINC00473 differential expression in LKB1-WT and LKB1-mutant cell lines was further corroborated by quantitative RT-PCR (qRT-PCR) data (Supplemental Figure 3B). Enhanced expression of a known LKB1 target gene, SIK1, was also confirmed but was not consistent across all cell lines tested (Supplemental Figure 3C). Moreover, SIK1 has relatively high basal expression. Additionally, we surveyed LINC00473 expression in 130 NSCLC cell lines using Affymetrix microarray data from the Cancer Cell Line Encyclopedia (CCLE) (37), and these arrays only contained the probes for LINC00473 tv1. We found that a subset of NSCLC cell lines showed outlier LINC00473 tv1 expression levels (Figure $1 \mathrm{H}$ and Supplemental Table 6). Analysis of cell lines with annotated LKB1 mutation status revealed that LINC00473 expression was significantly enhanced in LKB1-mutant NSCLC cell lines $(n=32)$ in comparison to LKB1-WT lines $(n=59)$ (Supplemental Table 6 and Figure 1I), and the enhancement was more significant as compared with the increase in SIK1 expression (Supplemental Figure 3D). Furthermore, two LKB1-WT cell lines with high LINC00473 expression, H292 and DV90, were predicted to have LKB1 loss based on a 16-gene signature score (35). H292 is a lung mucoepidermoid carcinoma cell line that contains a $t(11 ; 19)$ translocation that leads to the generation of CREB-regulated 
A

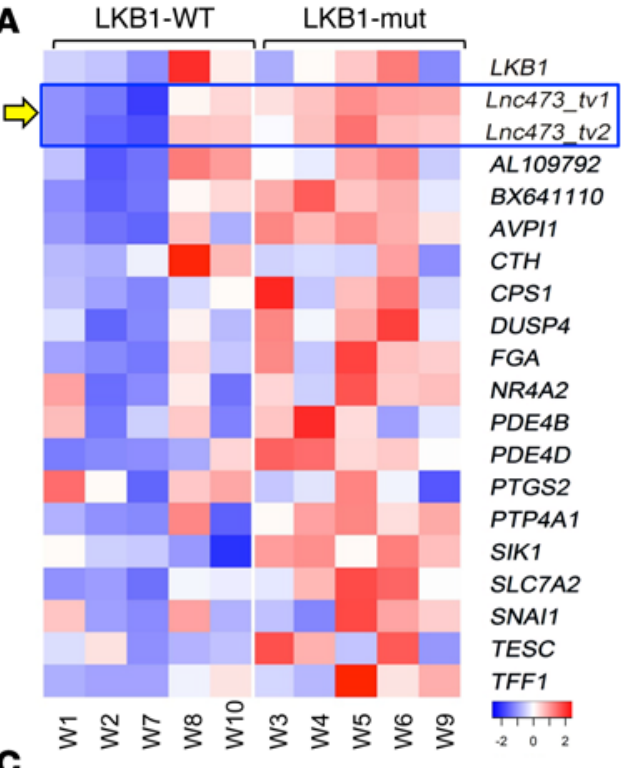

C
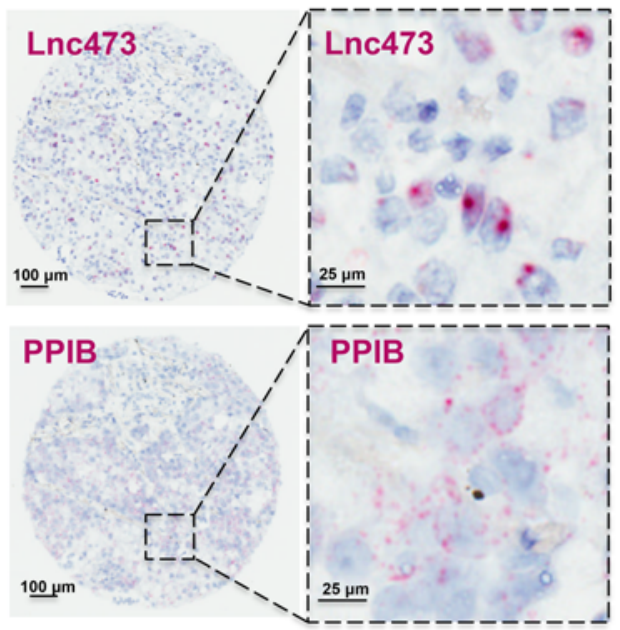

Lung adenocarcinoma (LKB1 mut, Lnc473 positive)

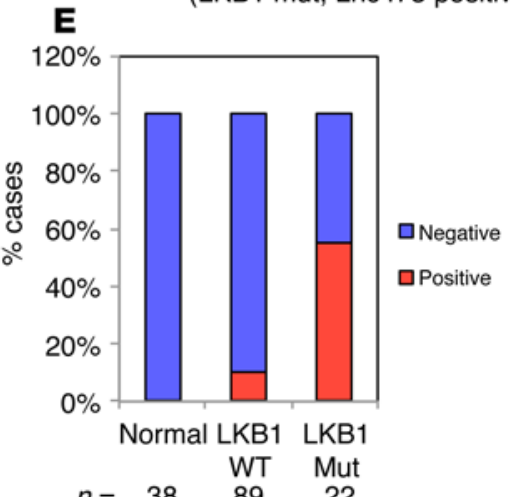

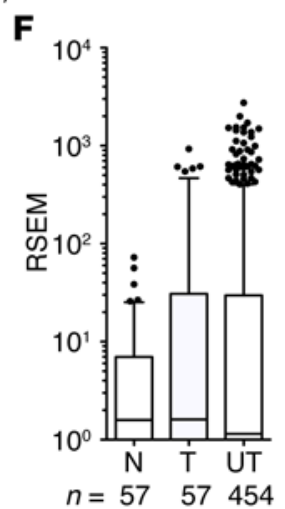
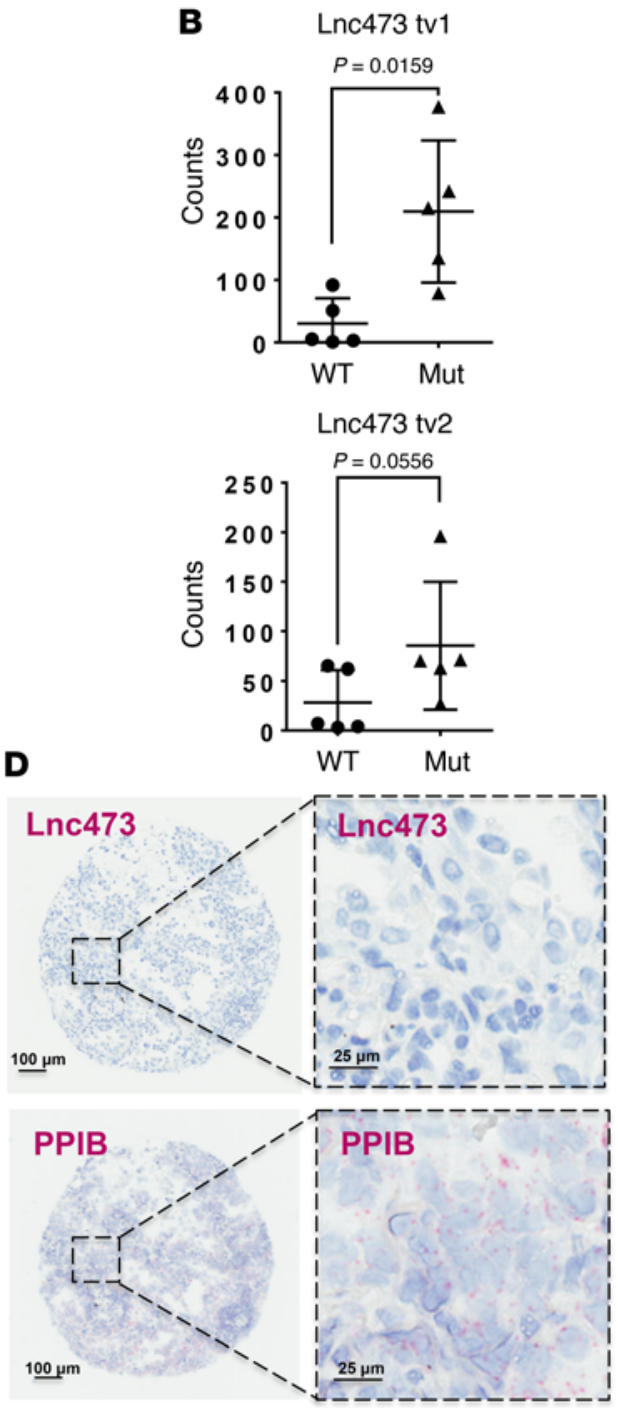

Lung adenocarcinoma

(LKB1 WT, Lnc473 negative)

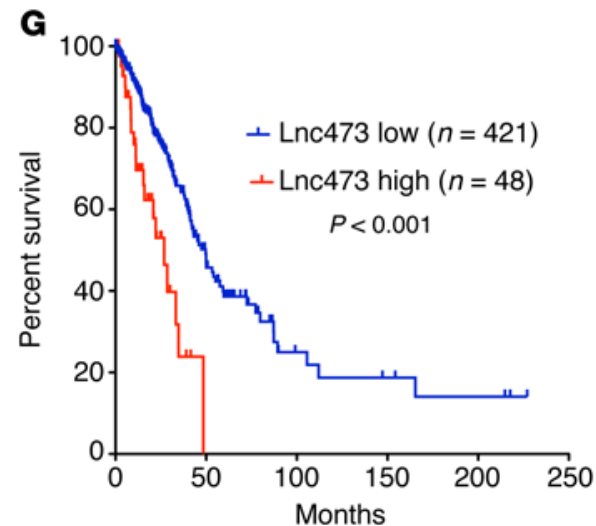

Figure 2. Enhanced LINC00473 expression is highly correlated with human lung adenocarcinoma with LKB1 mutational status and is associated with poor survival. (A) A heatmap shows gene expression levels in RNA samples isolated from 5 LKB1-WT and 5 LKB1-mutant FFPE LUAD samples in NanoString assays. (B) Expression of LINC00473 tv1, but not tv2, was significantly different between LKB1-WT and -mutant groups. (C and D) Representative images for tumors with LINC00473-positive (C) and -negative (D) signals based on RNAscope detection on FFPE LUAD sections. RNA ISH of the housekeeping gene PPIB was performed for sample RNA quality control. Scale bars: $100 \mu \mathrm{m}$ (left panels), $25 \mu \mathrm{m}$ (right panels). (E) A survey of human LUAD arrays indicated that $0 \%$ of normal lung tissues $(n=38), 10.11 \%$ of NSCLC tumors with annotated WT LKB1 $(n=89)$, and $55.54 \%$ of NSCLC tumors with annotated LKB1 mutations ( $n=22)$ were positive for LINC00473 expression. Only those tissues positive for the housekeeping gene PPIB expression were included in this analysis. LINCO0473 expression was positively correlated with $L K B 1$ mutations based on Fisher's exact test $\left(P=1.93 \times 10^{-5}\right)$. (F) TCCA-LUAD dataset showed outlier LINC00473 expression in matched tumor (T) $(n=57)$ compared with adjacent normal tissues $(N)(n=57)$ as well as unpaired tumor (UT) $(n=454)$. (C) Kaplan-Meier survival analysis of high LINC00473 expression $(n=48)$ and low LINC00473 expression $(n=421)$ in lung cancer patients $(P<0.001)$. See also Supplemental Figures $4-8$ and Supplemental Table 7. 

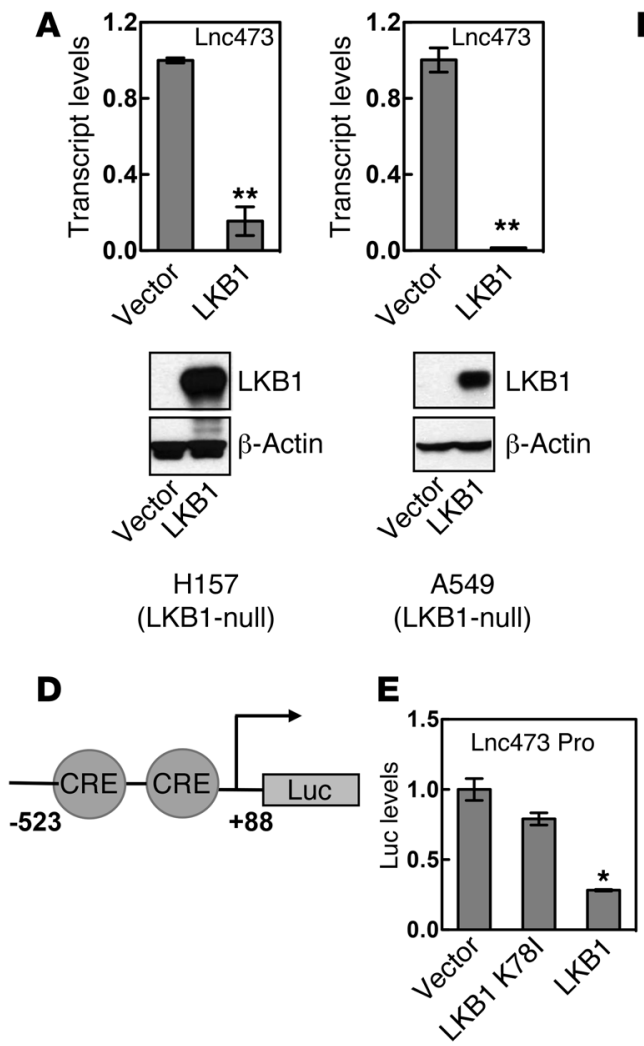
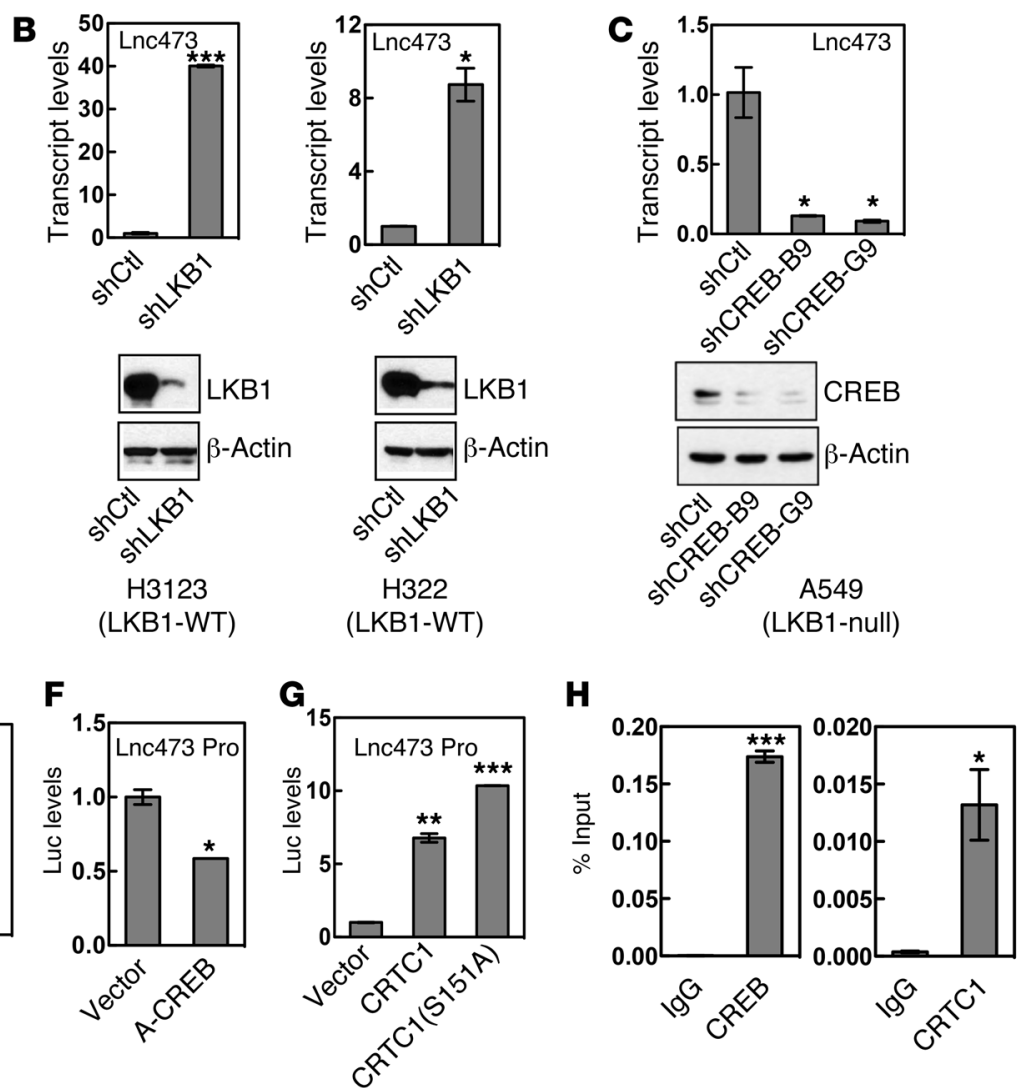

Figure 3. LINC00473 expression is regulated by the LKB1/CRTC1/CREB signaling axis. (A) qRT-PCR analysis showed that LINC00473 expression was significantly reduced in two LKB1-null NSCLC cell lines (H157 and A549) after transduction with LKB1 retroviruses (LKB1) for 96 hours, with cells transduced with vector retroviruses (CtI) used as controls. Western blotting confirmed LKB1 expression $\left(n=3,{ }^{* *} P<0.001\right)$. (B) LINCO0473 expression was enhanced upon LKB1 shRNA lentiviral infection in two LKB1-WT NSCLC cell lines (H3123 and H322) ( $n=3,{ }^{*} P<0.05$ and $\left.{ }^{* * *} P<0.0001\right)$. (C) LINC00473 expression was significantly reduced in A549 cells after transduction with two independent CREB shRNAs ( $\left.n=3,{ }^{*} P<0.05\right)$. (D) A schematic representation of the LINCO0473 promoter reporter. (E) Expression of LKB1, but not the kinase-dead K78I mutant in LKB1-null A549 cells caused significant repression of LINC00473 promoter reporter activity $\left(n=3,{ }^{*} P<0.05\right)$. (F) Expression of A-CREB in A549 cells significantly inhibited the LINC00473 promoter (Pro) activity ( $\left.n=3,{ }^{*} P<0.05\right)$. (G) Expression of WT or constitutively activated S151A CRTC1 increased LINC00473 promoter activity in LKB1-expressing H322 cells $(n=3$, ** $P<0.001$ and $\left.{ }^{* * *} P<0.0001\right)$. (H) ChIP analysis indicated that CREB and CRTC1 were significantly enriched on the LINC00473 promoter encompassing the CRE half sites in $A 549$ cells $\left(n=3,{ }^{*} P<0.05\right.$ and $\left.{ }^{* * *} P<0.0001\right)$. See also Supplemental Figure 9.

transcription coactivator-MAML2 (CRTC1-MAML2) fusion (38) and subsequent constitutive activation of cyclic AMP-responsive element-binding protein-mediated (CREB-mediated) transcription, thus mimicking LKB1 loss. Collectively, these data strongly support that LINCOO473 is consistently the most elevated gene in LKB1-inactivated NSCLC cell lines regardless of other coexisting gene mutations.

Direct RNA detection in FFPE specimens revealed that LINCOO473 expression is elevated in a subset of NSCLCs that tend to have mutations in the LKB1 gene coding regions. To investigate whether LINC00473 could serve as a potential biomarker for LKB1 status in human NSCLC cancers, we evaluated expression of LINC00473 and several other LKB1 targets in formalin-fixed, paraffin-embedded (FFPE) human lung adenocarcinoma (LUAD) specimens. Target amplification-based strategies such as RT-PCR and microarray analyses for measuring RNA levels from fixed tissue is complicated by the fact that the RNA is highly cross-linked and significantly fragmented. NanoString-based assays are optimal for gene expression quantification using FFPE tumor-derived RNA samples, since the barcoded fluorescent probes recognize small target regions ( $100 \mathrm{bp})$, allowing direct single-molecule counting without the need for target amplification (34). Therefore, we performed NanoString assays for FFPE tumor-derived RNAs from 5 LKB1-WT and 5 LKB1-mutant human LUAD specimens. The LKB1 gene mutation status was analyzed by exon sequencing as previously described (19), and the results are shown in Supplemental Table 7. LINC00473 expression was consistently found to best correlate with tumor LKB1 status among all the genes tested (Figure 2A). Expression of LINC00473 tv1, but not tv2, was significantly enhanced in LUAD with LKB1 mutations (Figure 2B). These data suggest that expression of LINC00473 tv1 alone can predict tumor LKB1 status.

To examine whether LINC00473 expression could be directly visualized at the cellular level in human FFPE tumors, we next performed RNA in situ hybridizations (RNA ISH) for detection of LINC00473 transcripts using customized LINC00473 probes (RNAscope). The specificity of LINC00473 probes was first validated by positive LINC00473 signals in LKB1-null A549 xenograft tumors and negative signals in LKB1-WT H522 xenograft tumors (Supplemental Figure 4, A and B). LINC00473 

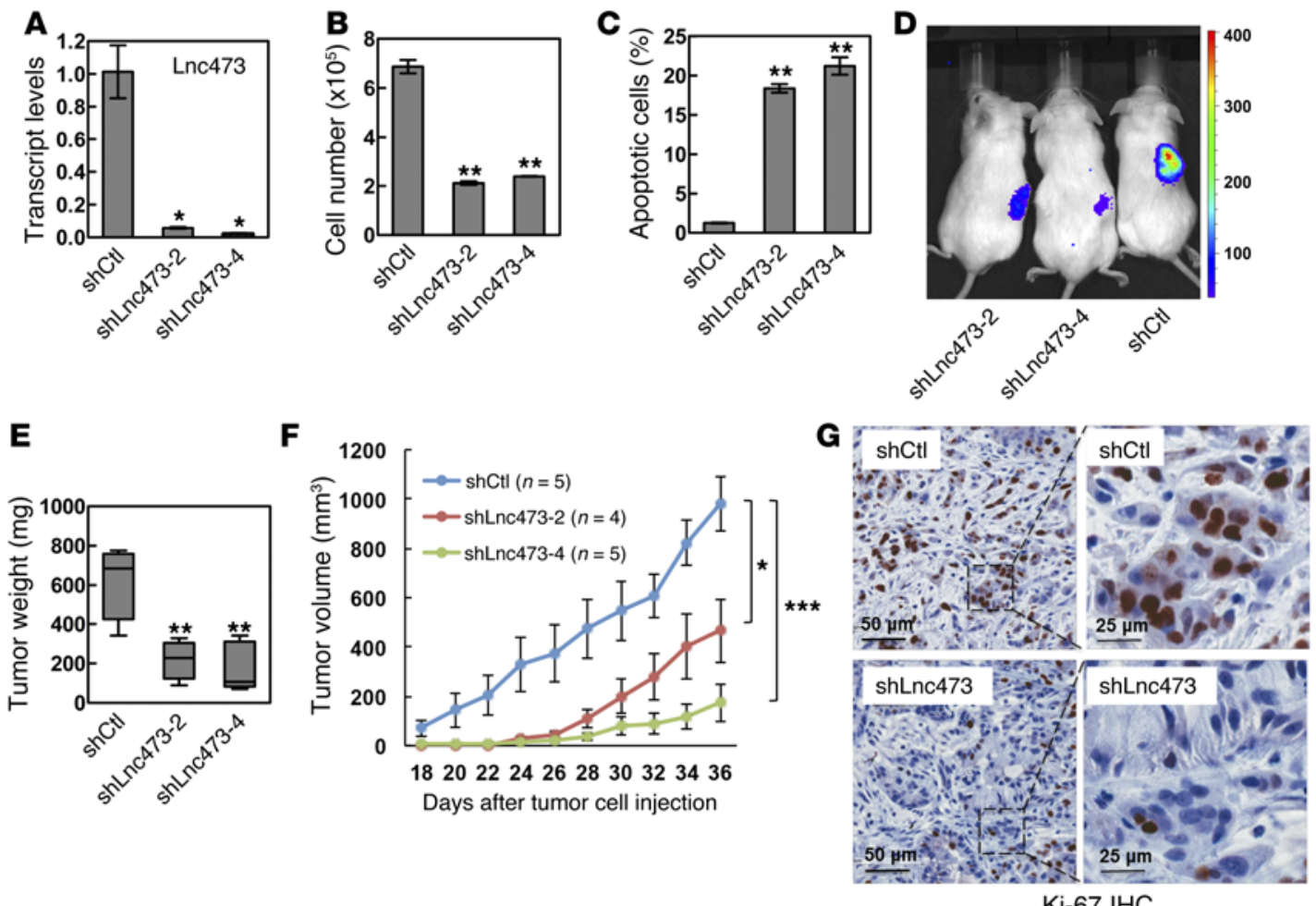

$\mathrm{Ki}-67 \mathrm{IHC}$

Figure 4. Depletion of LINC00473 expression in LKB1-null NSCLC cells causes reduced cell growth and survival in vitro and in vivo. (A) Luciferaseexpressing A549 cells were infected with one of two lentiviral-based LINC00473 shRNAs or the scrambled shRNA control (shCtl). Transduced cells were harvested 96 hours later, and LINC00473 expression was quantified by qRT-PCR ( $n=3,{ }^{*} P<0.05$ ). (B and C) Transduced cells at 96 hours after transduction were cultured at $2 \times 10^{5}$ per well in 6-well plates for another 96 hours, and viable cell numbers were measured using a trypan blue assay (B), and apoptotic cells were detected by Annexin V/PI staining (C) $\left(n=3,{ }^{* *} P<0.001\right)$. (D-F) A total of $1 \times 10^{6} \mathrm{~A} 549$-luc cells after transduction with shLINC00473 or shCtI for 72 hours were injected subcutaneously into the dorsal flanks of NOD/SCID mice. Representative bioluminescent images of mice injected with shCtI or shLINC00473 A549-luc cells (D) and the weights of excised tumors (E) at the end points are shown. Tumor growth is shown at different days after tumor cell injection (F). $\left({ }^{*} P<0.05,{ }^{* *} P<0.001,{ }^{* * *} P<0.0001\right)$. (G) Immunohistochemical staining of A549-control and A549-shLINC00473 xenograft tumor sections with Ki-67 antibody. See also Supplemental Figures 10-11. Scale bars: $50 \mu \mathrm{m}$ (left panels), $25 \mu \mathrm{m}$ (right panels).

transcripts were detected as easily distinguishable nuclear "dots" in A549 xenograft tumors (Supplemental Figure 4, A and C). We then performed RNA ISH on an FFPE human LUAD tissue array. This array also included FFPE cell pellets from LKB1-null A549 and LKB1-positive $\mathrm{H} 322$ as controls, which showed positive and negative LINC00473 signals, respectively (Supplemental Figure 4, D and E). We analyzed only those tumors that were positive for the housekeeping gene peptidylprolyl isomerase (PPIB), indicative of tumors with good RNA quality. Representative positive and negative LINC00473 staining results are shown (Figure 2, $\mathrm{C}$ and D). We found that all normal human lung tissues $(n=38)$ were negative for LINC00473 staining while exhibiting positive staining for PPIB staining (Figure 2E and Supplemental Figure $4 \mathrm{~F}$ ), indicating absent or low basal LINC00473 expression in normal lung tissues. A total of 9 of 89 NSCLC specimens $(10.11 \%)$ with annotated LKB1-WT and 12 of 22 lung tumors (54.54\%) with annotated LKB1 mutations were positive for LINC00473 staining (Figure 2E and Supplemental Figure 4, G-J). LINC00473 expression showed significant positive correlation with LKB1 mutations based on Fisher's exact test $\left(P=1.93 \times 10^{-5}\right)$. It is likely that those tumors carrying the LKB1-WT gene yet showing elevated LINC00473 expression have LKB1 functional inactivation due to other mechanisms besides LKB1 mutations, such as epigenetic silencing, posttranslational modifications, or alteration in LKB1 signaling components (20-22). On the other hand, it is worth noting that not all mutations found in LKB1 are inactivating. Tumors carrying the LKB1 gene mutations yet not showing LINC00473 induction could have intact LKB1 function if the mutations do not impair LKB1 function. For example, one of the tumors with LKB1 F354L mutation, which was predicted not to have damaging effects on LKB1 function based on PolyPhen-2 prediction (39), showed undetectable LINC00473 expression. In addition, negative LINC00473 staining was found in several other cancer types and tissues, including prostate cancer, invasive ductal carcinoma (IDC) breast, large cell lymphoma, hepatocellular carcinoma, colon adenocarcinoma, and osteosarcoma, as well as placenta, tonsil, and normal spleen ( $n=1$; data not shown). These data strongly indicate that LINC00473 is low or undetectable in normal lung tissues but exhibits elevated expression in the subset of lung NSCLC specifically with functional LKB1 inactivation, indicating that LINC00473 is a potential robust surrogate biomarker for LKB1 functional status in lung cancer.

Elevated LINCOO473 expression is associated with tumor LKB1 mutations and correlated with poor prognosis in TCGA lung adenocarcinomas. To investigate any potential association among LINC00473 expression, LKB1 mutation status, and clinical lung 
cancer data, we examined a LUAD RNAseq dataset from The Cancer Genome Atlas (TCGA) (40). We observed a subset of lung cancers with outlier LINC00473 expression (90th percentile rank) and a significant difference in LINC00473 expression between tumors (either paired, $n=57$; or unpaired, $n=454$ ) and normal tissues $(n=57)$ (Figure 2F). LUAD samples with high LINC00473 expression were enriched with LKB1 gene-level nonsynonymous somatic mutations, including small insertions or deletions (INDELs) within the LKB1 gene coding regions (Supplemental Figure 5). LINC00473 expression was not associated with KRAS and TP53 gene mutations (Supplemental Figure 5), which are two well-known somatic mutations that can occur concurrently with LKB1 loss. Moreover, the difference in LINC00473 expression between LKB1-WT and LKB1-mutant populations was more significant compared with SIK1 or LKB1 expression (Supplemental Figure 6). LINC00473 expression was positively correlated with LKB1-loss gene signature (35) and inversely correlated with LKB1 expression, and such correlations were more significant in comparison to SIK1 expression and LKB1 expression (Supplemental Figure 7). These data support a strong association of LINC00473 expression with the LKB1 inactivation in LUAD samples.

Kaplan-Meier survival analysis showed highly significant difference in overall survival between the high expression $(n=48)$ and low expression $(n=421)$ groups $(P<0.001$, Figure $2 G)$. The elevated LINC00473 expression significantly correlated with a shorter survival time in LUAD patients ( $<50$ months). When analyzing those tumors with the available data on LINC00473 expression, LKB1 mutations, and the clinical information, we observed that LKB1 mutation status was not significantly associated with survival, but high LINC00473 expression was associated with poor survival within both the LKB1-WT and -mutant groups (Supplemental Figure 8). These data indicate that LINCO0473 has a prognostic value and may play an important role in cancer progression.

LINC00473 expression is promoted by LKB1 loss-induced CRTC/ CREB activation. High LINC00473 expression has a positive correlation with LKB1 functional inactivation in NSCLC cell lines and human primary tumors, suggesting that LKB1 inactivation leads to increased expression of LINC00473. To further examine LKB1 regulation of LINC00473 expression, we first tested whether cellular LKB1 levels directly impacted LINC00473 expression. We observed that introduction of exogenous LKB1 in LKB1-null cancer cell lines (H157, A549) resulted in a significant decrease in LINC00473 transcript level (Figure 3A), whereas shRNA-mediated depletion of endogenous LKB1 expression in LKB1-positive cells (H3123, H322) led to an increase in LINC00473 levels (Figure 3B). Therefore, modulating cellular LKB1 protein expression affects LINC00473 expression in NSCLC cells.

LKB1 regulates multiple downstream AMPK family members, influencing multiple signaling pathways (5). We previously showed that loss of LKB1 expression resulted in dephosphorylation and nuclear entry of CRTC transcriptional coactivators and subsequent CREB-mediated transcriptional activation in both lung and esophageal cancer cells $(41,42)$. LINC00473 was transiently upregulated in response to cAMP signaling in human ocular ciliary smooth muscle cells (43). The LINC00473 gene contains two cAMP-responsive element (CRE) half sites within the proximal promoter region. To test whether the loss of LKB1 induces CRTC/CREB activation and promotes LINC00473 expression, we first depleted CREB using lentiviral-mediated shRNAs or expressed a dominant negative form of CRTC (dnCRTC) that interferes with CRTC/CREB interaction. We detected a reduction in the LINC00473 transcript level in CREB-depleted or dnCRTC-expressing A549 cells (Figure 3C and Supplemental Figure 9). LKB1 overexpression further blocked LINC00473 expression (Supplemental Figure 9). These data demonstrate that LINC00473 is regulated by LKB1 loss and CRTC/CREB activation. Next, we cloned the proximal LINC00473 promoter sequence $(-523$ to +88$)$ encompassing two CRE sites into the upstream region of a luciferase reporter (pGL3 basic) (Figure 3D) and then determined LINC00473 promoter activity by modulating LKB1/CRTC/CREB signaling. We observed that the LINCO0473 promoter reporter was significantly repressed by overexpression of LKB1, but not the LKB1 kinasedead mutant K78I, when transfected in LKB1-deficient A549 cells (Figure 3E). Moreover, promoter activity was significantly inhibited by expression of A-CREB (Figure 3F), a dominant-negative mutant that specifically blocked CREB binding to DNA $(44,45)$. Also, the LINCO0473 promoter reporter was activated by overexpression of CRTC1 and, to a greater extent, by a constitutively activated form of CRTC1 (S151A) (Figure 3G). Finally, chromatin immunoprecipitation (ChIP) assays demonstrated that CRTC1 and CREB were enriched in the LINCOO473 promoter region spanning the CRE sites (Figure 3H). Overall, these data suggest that LINC00473 transcription is directly induced by CRTC/ CREB activation in LKB1-inactivated NSCLC cells.

In vitro and in vivo approaches revealed critical functions of LINCO0473 in the growth of LKB1-null lung cancer cells. High LINC00473 expression correlated with poor survival of lung cancer patients (Figure 2G), indicating a role for LINC00473 in cancer progression. Therefore, we investigated the functional significance of sustained LINC00473 expression in LKB1inactivated NSCLC cells. We first determined the functional impact of LINC00473 depletion on cell growth and survival using two independent lentiviral pLKO.1-based shRNAs targeting exon 2 of LINC00473 tv1 (shLnc473-2 and shLnc473-4). We found that two shLnc 473 caused an approximately $90 \%$ reduction in LINC00473 transcript levels in A549 cells at 96 hours after lentiviral infection (Figure 4A). The shLnc473-expressing as well as scrambled shRNA control-expressing cells were subsequently assayed for cell growth and survival. LINC00473 knockdown reduced cell proliferation and enhanced apoptosis in LKB1-null A549 cells (Figure 4, B and C). Similar effects of LINC00473 depletion on the LKB1-null NSCLC cell line H157 were also observed (Supplemental Figure 10). Conversely, exogenous LINC00473 overexpression via retroviral transduction in LKB1-WT H522 lung cancer cells resulted in a moderate, yet significant increase in cell proliferation (Supplemental Figure 11, A and B). These data demonstrate that LINC00473 is essential for maintaining LKB1-inactivated lung cancer cell growth and survival.

We next determined the effect of LINC00473 depletion on the growth of NSCLC xenografts. The luciferase-expressing A549 (A549-luc) cells were transduced with lentiviral-based shLnc473 or scrambled shRNA control for 72 hours, and then equal numbers of LINC00473-depleted and control cells were 
implanted into NOD/SCID mice by subcutaneous injection. We observed that LINC00473 depletion significantly reduced tumor size and weight, and blocked the growth of A549-luc xenografts over time (Figure 4, D-F). IHC analysis showed that LINC00473-depleted xenograft tumors contained a reduced number of cells that were positive for the cell proliferation marker Ki-67 (Figure 4G). Similarly, depletion of LINC00473 expression decreased the growth of $\mathrm{H} 157$ xenografts in NOD/ SCID mice (Supplemental Figure 10, D-F). Therefore, both in vitro and in vivo evidence supports the critical functions of LINC00473 in regulating lung cancer growth and survival.

LINCO0473 is a nuclear IncRNA and functions as a regulator of gene expression in part through interacting with NONO and modulating CRTC/CREB transcription. The molecular mechanisms underlying LINC00473 functions are unknown. Studies have indicated that lncRNAs may be involved in various processes, including transcription, splicing, posttranscriptional regulation, organization of protein complexes, cell-cell signaling, and allosteric regulation of proteins (46). Knowledge of subcellular localization of lncRNAs can provide a clue about lncRNA functions. We observed nuclear localization of LINC00473 transcripts in FFPE human lung cancer specimens using customized LINC00473 probes in RNAscope RNA ISH assays (Figure 2C). To validate LINC00473 as a nuclear lncRNA, we performed a subcellular fractionation assay and RNA fluorescence in situ hybridization (RNA-FISH). For the fractionation assay, we prepared cytoplasmic and nuclear fractions and determined LINC00473 transcript levels in both fractions. We confirmed that in comparison to the respective cytoplasmic (tRNA) and nuclear (U6) controls, LINC00473 is enriched in the nuclear fraction (Figure 5, A and B). For RNA-FISH, we hybridized fixed cells with a mixture of 27 oligonucleotide probes (20-mer) targeting LINC00473, with each probe linked with a single Quasar 570 fluorophore. We observed positive nuclear signals with 1 to 2 distinctive dot-like structures, as well as less intense, diffuse signals outside the dots (Figure 5C). LINC00473 signals were undetectable when hybridizations were performed in the presence of RNase (Supplemental Figure 12A), indicating that the signal detection was RNA dependent. Moreover, exogenous LINC00473 showed similar nuclear localization when overexpressed in LKB1WT H522 cells (Supplemental Figure 12, B and C). These data strongly support that LINC00473 has distinct nuclear localization and likely participates in nuclear functions.

To investigate LINC00473-interacting proteins, we performed an RNA pull-down assay followed by a proteomic analysis of the LINC00473-associated protein complex in A549 cells. We incubated the in vitro transcribed LINC00473 bound to beads with A549 nuclear extracts to purify the LINC00473 RNA-protein complex. The LINC00473-associated protein complex components were separated by SDS-PAGE (Figure 6A), and the protein identity was revealed by mass spectrometry (MS). A notable protein was a known CRTC-interacting protein, NONO (47), with 115 peptides detected in this MS analysis (Supplemental Table 8). To validate the physical interaction between LINC00473 and NONO, we performed RNA pull-down followed by Western blotting with NONO antibodies. NONO was readily detected in the LINCO0473 RNA pull-down complex but not in the control samples, including LINC00473 antisense RNA, lncRNA MEG3, and beads only (Fig- ure 6B). We also performed RNA immunoprecipitation (RNA-IP) for the RNA-NONO complex using NONO antibodies and measured the amount of LINCO0473 associated with NONO immunoprecipitates. The immunoprecipitated NONO protein levels were confirmed by Western blotting (Figure 6C). The qRT-PCR results showed significant enrichment of LINC00473, but not the negative control, ASNS, in NONO immunoprecipitates (Figure 6D). These data indicate that NONO is an LINC00473-associated protein.

NONO interacts with CRTC coactivators upon cAMP stimulation, which is essential for CREB-mediated transcription (47). Since LINC00473 physically interacted with NONO, we hypothesize that LINC00473 regulates NONO recruitment to CRTCs and promotes subsequent activation of CREB target gene transcription. To test this hypothesis, we determined the interaction of NONO and CRTC when LINC00473 was either depleted or overexpressed using mammalian two-hybrid assays. We observed that depletion of LINC00473 caused a reduced interaction of Gal4-NONO and CRTC1, which was indicated by reduced activation of a Gal4 promoter reporter (Figure 6E). Conversely, enhanced LINC00473 expression promoted NONO-CRTC1 interaction (Figure 6F). These data suggest that LINC00473 facilitates the recruitment of NONO to CRTC and subsequently promotes CREB-mediated transcription. To further examine whether LINC00473 regulates endogenous CRTC/CREB target genes, we performed gene expression analysis in lentiviral-based scrambled shRNA control and LINC00473-depleted A549 cells, as well as in retroviral-based vector control and LKB1-expressing A549 cells in NanoString assays. LINC00473 depletion impaired expression of several known and potential CRTC/CREB targets, including CTH, CPS1, DUSP4, FGA, NR4A2, PDE4B, PDE4D, PTGS2, PTP4A1, SIK1, SLC7A2, TESC, and TFF1, expression of which was induced by LKB1 loss (Figure 6G). Moreover, NEDD9, a CRTC/CREB target gene implicated in regulating cell proliferation and metastasis (48), was downregulated in LINC00473depleted A549 cells, although its expression was not significantly different between LKB1-mutant and -WT cell lines (Supplemental Figure 13). Furthermore, qRT-PCR analysis showed that expression of exogenous LINC00473 in LKB1-WT H522 significantly promoted transcription of several CREB target genes, such as CPS1, PDE4B, PTGS2, and PDE4D (Supplemental Figure 11C). All these data support a model whereby LINC00473 acts as a coactivator with CRTC/CREB in a positive feedback mechanism to maintain high steady-state levels and induce expression of other LKB1-regulated targets (Figure 7).

\section{Discussion}

Lung cancer is the leading cause of cancer death. Progress in lung cancer treatment will require improved predictive biomarkers so that cancer patients can be provided with the most effective treatments available, as well as a larger repertoire of therapeutic targets. In this study, we discovered an lncRNA (LINC00473) whose elevated expression was highly associated with loss function of the tumor suppressor $L K B 1$ gene, one of the most common mutational events in lung cancer. Our gene expression and functional data strongly support the potential utility of LINC00473 as a biomarker and as a therapeutic target for lung cancers with impaired LKB1 signaling. Moreover, 


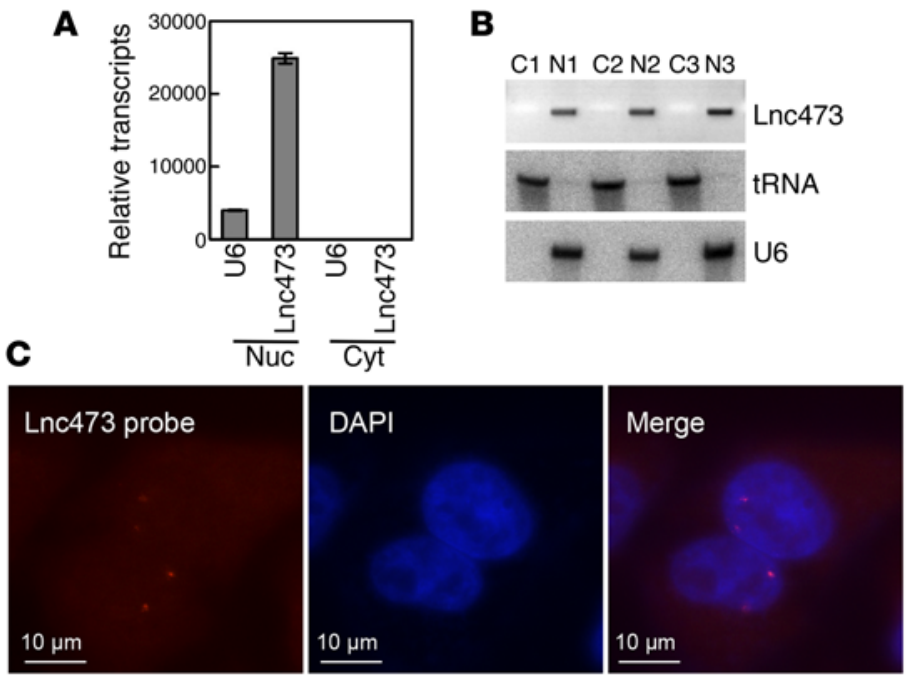

Figure 5. LINC00473 shows predominantly nuclear localization with distinct nuclear structures. (A) Transcript levels of LINC00473 and U6 (a nuclear marker) in the nuclear (Nuc) and cytoplasmic (Cyt) fractions obtained from A549 cells were quantified by qRT-PCR assays. (B) LINC00473 transcripts were enriched in the nuclear compartment when compared with nuclear marker U6 and cytoplasmic marker tRNA by Northern blot analysis in three separate nuclear (N) and cytoplasmic (C) fractions obtained from A549 cells. (C) Nuclear localization of LINC00473 was detected by RNA-FISH in A549 cells. LINC00473 RNA-FISH probe sets were labeled with Quasar 570 dyes (red), and nuclei were labeled with the DNA dye DAPI (blue). Scale bars: $10 \mu \mathrm{m}$. See also Supplemental Figure 12 .

we provide mechanistic insights into LINC00473 as a critical nuclear regulator of gene expression in lung cancer.

Recent studies reveal that specific lncRNA expression is associated with disease state $(27,49)$, strongly supporting the utility of lncRNAs in clinical diagnosis and prognosis. Loss of LKB1 function in NSCLC cells caused differential responses to therapeutic agents in vitro and in animal studies. LKB1 inactivation sensitized NSCLC cells to the metabolism drug phenformin and a COX2 inhibitor while conferring resistance to PI3K/Akt and MEK inhibitors (14-17). However, no specific effective treatments are currently available for patients with LKB1-deficient lung cancer. One big barrier is the lack of a reliable assessment for tumor LKB1 inactivation that can be used in clinical trials for patient selection and treatment evaluation. Current clinical LKB1 analysis includes evaluation of LKB1 mutations through sequencing 9 coding exons and flanking region of the $L K B 1$ gene as well as IHC assay of LKB1 protein expression. However, LKB1 functional inactivation could result from mutations across the entire $L K B 1$ gene, epigenetic silencing, or posttranslational inactivation $(20,22,50)$, posing a great challenge for detection of LKB1 functional loss through direct genomic sequencing. Also, not all LKB1 mutations impair LKB1 functions. IHC assay was shown to be a means to detect LKB1 protein loss (51); however, the LKB1 antibodies used in the study were not highly specific. Recently, a 16-gene signature was reported to be capable of predicting tumor cells with LKB1 inactivation (35). Yet expression of those individual genes is not completely correlated with the tumor LKB1 status, and combined scoring of multiple genes for individual tumors requires complicated analysis.
In this study, we observed that LINCOO473 is consistently the most highly induced gene in LKB1-inactivated primary NSCLC samples and derived cell lines, supporting the notion that LINC00473 expression could be used for predicting LKB1 functional status in clinically relevant FFPE tumor specimens. There are advantages in using LINC00473 as a surrogate marker for tumor $L K B 1$ functional inactivation. First, LINC00473 expression is a functional readout for LKB1 inactivation; thus, LINC00473-based detection will be advantageous relative to direct sequencing of the entire $L K B 1$ gene or IHC analysis for the detection of LKB1 protein. We observed that a subset of human lung tumors without detectable mutations in the coding region of the $L K B 1$ gene showed positive staining for LINC00473. These tumors likely reflect scenarios where functional $L K B 1$ inactivation is caused by epigenetic effects such as promoter hypermethylation or functional suppression by other mechanisms, including alterations in other components of the LKB1 pathway. For example, BRAF activating mutations were shown to suppress LKB1 kinase activity and thus inactivated LKB1 signaling (20). On the other hand, a subset of tumors with LKB1 mutations had non-detectable LINC00473 expression. One possibility is that these tumors have $L K B 1$ mutations yet such mutations have no significant impact on LKB1 functions. Also, tumor cells having the mutation(s) on one allele of the LKB1 gene may express sufficient levels of functional LKB1 proteins. Second, the presence of a significant number of LKB1-WT stromal cells within tumors can obscure detection of LKB1-null tumor cells in LKB1 IHC studies. Since LINC00473 is expressed at low or undetectable levels under normal conditions but at high levels in LKB-inactivated cells, detection of upregulated LINC00473 expression will not be affected by the presence of stromal cells. Third, LINC00473 has sufficiently high expression in LKB1-inactivated lung cancer and can be detected in biopsy specimens in the clinic. We present proof-of-concept data showing that both NanoString-based expression assay and RNAscope-based RNA ISH can be used for LINC00473 detection in clinically relevant FFPE tumors. The NanoString-based assay is rapid and reproducible in clinical laboratories, and provides quantitative expression scores. The RNAscope-based assays, while requiring subjective scoring due to variable levels of staining signals, can allow colorimetric detection of LINC00473-expressing tumor cells to infer LKB1 functional status. Such transcript-based tests, capable of detecting pathway activity, are of special importance for tumor suppressor gene detection, as histologically clinical diagnosis based on LKB1 IHC and DNA mutation assays are more difficult as compared with activated oncogene detection. Our data suggest the potential to develop a LINC00473 expression-based test for scoring tumors with LKB1 functional loss that could be integrated with clinical trial design for patient selection and treatment decisions. In the future, it will be important to identify the specific mechanisms underlying $L K B 1$ silencing in the subset of "LKB1-WT, LINC00473-positive" tumors or intact functional LKB1 signaling in "LKB1-mutant, LINC00473-negative" tumors. Due to the complexity of working with stored primary tumors with heterogeneous stromal contamination, this effort will require extensive follow-up studies using fresh samples at the genomic, epigenomic, and expressional levels. 
A

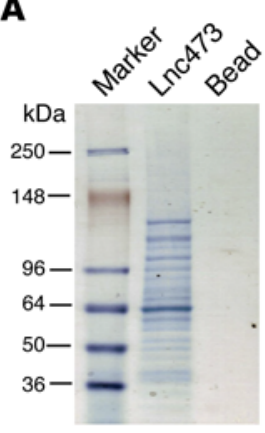

E

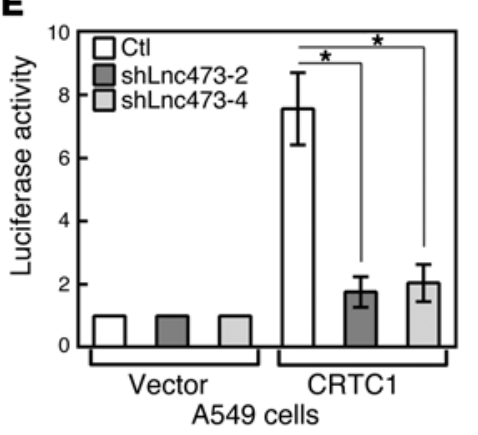

C

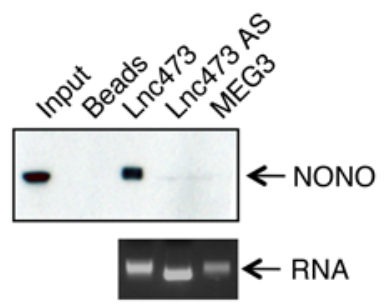

RNA

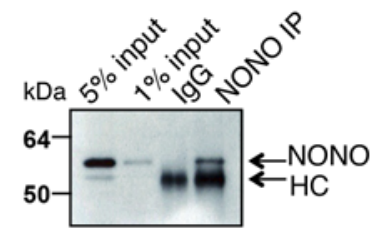

$\mathbf{F}$

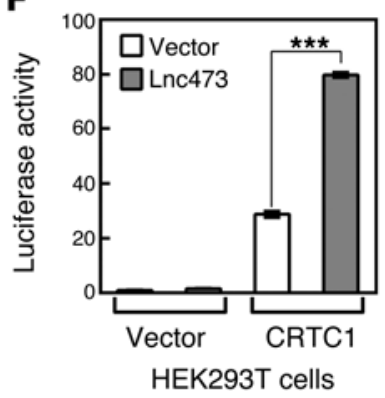

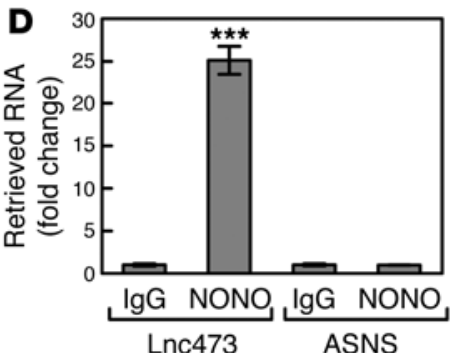

G

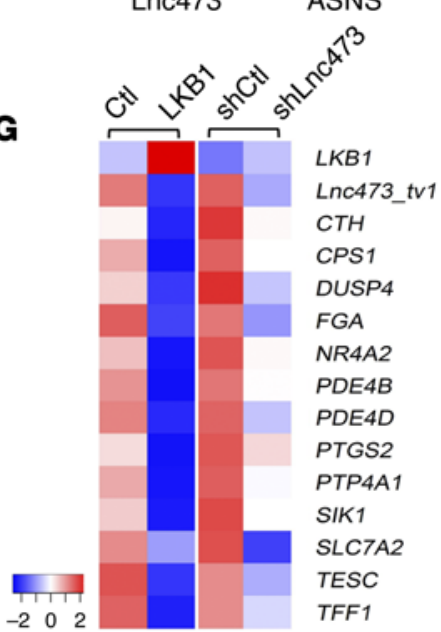

Figure 6. LINC00473 is associated with NONO protein and stimulates CRTC-NONO interaction. (A) Coomassie blue staining of the LINC00473-associated proteins by RNA pull-down in A549 cells. (B) Specific association of LINC00473 RNA with NONO protein was validated through RNA pull-down followed by Western blot analysis. LINCO0473 antisense and MEG RNA were used as controls. (C) Immunoprecipitation of endogenous NONO protein was validated via Western blotting (HC, heavy chain). (D) LINC00473 was significantly enriched in NONO immunoprecipitates relative to the IgC control by qRT-PCR assay. ASNS was used as a negative control $\left(n=3,{ }^{* *} P<0.0001\right)$. (E) Depletion of LINC00473 caused reduced CRTC1-NONO interaction. A549 cells, after transduction with LINC00473 shRNA (shLINC00473-2 and -4) or the scramble shRNA lentiviruses (Ctl) for 72 hours, were cotransfected with Gal4-NONO, pSG5-luc (a firefly luciferase reporter containing Gal4-binding sites), pEF-RL (Renilla luciferase), as well as vector control or CRTC1. The luciferase activity was measured 24 hours after transfection $\left(n=3,{ }^{*} P<0.05\right)$. (F) Overexpression of LINC00473 enhanced CRTC1-NONO interaction. HEK293T cells were transfected with Gal4-NONO, CRTC1, pSC5-luc, or pEF-RL in the presence of vector or LINC00473 construct. The luciferase activity was determined at 24 hours after transfection ( $n=3$, $\left.{ }^{* * *} P<0.0001\right)$. (C) Relative expression levels of LKB1-regulated genes in shLINC00473-versus shCtl-expressing A549 cells and in LKB1 versus vector control A549 cells showed that LINC00473 depletion attenuated some common target gene expression induced by LKB1 loss. See also Supplemental Figure 13.

Patients with high LINC00473-expressing lung cancers had shorter survival times, suggesting that LINC00473 likely confers lung cancer cells with aggressive behaviors. Interestingly, a substantial number of lncRNAs in the human genome seem to have arisen within the primate lineage based on sequence conservation (52), and LINC00473 belongs to this group. It is unclear whether LINC00473 may contribute to any unique features associated with human cancers. Our functional data strongly indicate an essential role for LINC00473 in maintaining human NSCLC cell growth and survival. LINC00473 has low or undetectable expression in normal tissues; therefore, targeting LINC00473 expression is an attractive approach for specifically blocking lung cancer without significantly affecting normal tissues. Currently, anticancer drugs mainly target DNA or proteins in tumor cells. Therapeutic development for RNA-based targeting is in its infancy, but various new approaches are being explored, such as antisense oligonucleotides and RNAi. Progress has been made to improve delivery and specificity. For example, first proof-ofprinciple RNAi therapeutics based on RNAi and lipid nanoparticles (LNPs) has been tested in humans (53), supporting further development of RNA-targeting drugs in treating cancers. There- fore, approaches for targeting LINC00473 expression for blocking NSCLC should be further investigated.

We identified an upstream LKB1/CRTC/CREB signaling axis directly controlling LINC00473 transcription. LINC00473 expression is strongly tied with the LKB1 signaling in different cellular contexts, as we observed that upregulation of LINC00473 is tightly linked with LKB1 functional status in spite of many other gene mutations coexisting in cancer cells. Future detailed analysis of the LINC00473 promoter/enhancer is necessary to gain insights into the tight, specific regulation by the LKB1 pathway. LINC00473 is a nuclear IncRNA, and the distinct nuclear pattern of LINC00473 is intriguing. Mechanistically, LINC00473 promotes the recruitment of NONO to CRTCs and enhances transcriptional activation of CREB-mediated transcription. Our data thus identified a function for LINC00473 in assisting with the recruitment of critical factors to assemble active CRTC/CREB transcription complexes and promote transcription, elucidating a potential positive feedback mechanism for LINC00473 in regulating gene expression. These data are consistent with the finding that LINC00473 depletion reduced the expression of a set of genes that are induced by LKB1 loss, suggesting that LINC00473 likely mediates certain effects of the loss of LKB1 


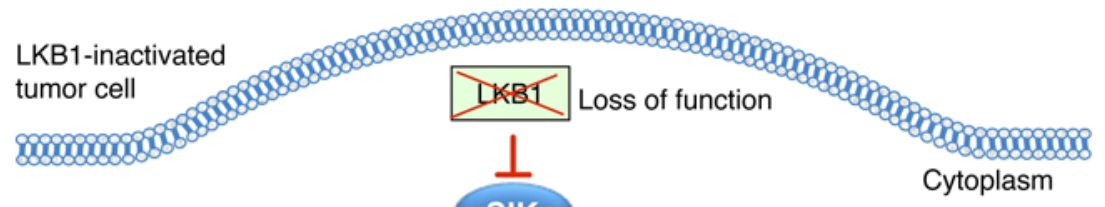

SIK

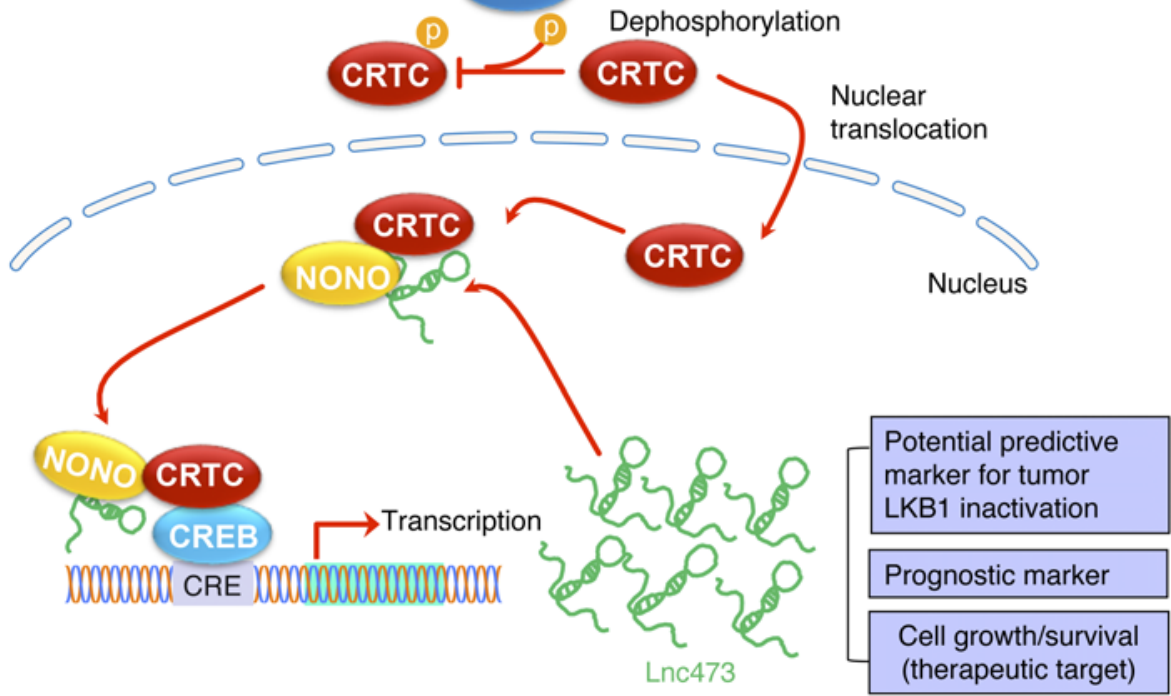

Figure 7. A model for the molecular basis of LINC00473 induction and the role of sustained LINC00473 expression as a potential biomarker and prognostic marker, therapeutic target, and gene regulator for LKB1-inactivated NSCLC.

tumor suppression. It should be noted that LINC00473 appeared to have a negative feedback regulation in normal cells after cAMP stimulation, as LINC00473 RNAi caused moderate transcriptional upregulation of cAMP signaling-responsive genes in response to an EP2 agonist (43). The molecular action of LINC00473 in regulating CAMP/CREB signaling should be further investigated and compared between normal cells with low or undetectable basal LINC00473 expression and cancer cells with sustained high LINC00473 expression. Finally, LINC00473 most likely has other mechanisms that contribute to its regulatory functions. Elucidation of the endogenous chromatin-associated LINC00473 binding sites as well as downstream target genes will provide mechanistic insights into the roles of this lncRNA in lung tumorigenesis.

In summary, we identified a nuclear lncRNA LINC00473 whose expression has potential utility in classifying LKB1 functional status in human NSCLC and is associated with poor patient survival. Sustained LINC00473 expression is essential for maintaining NSCLC cell growth and survival, supporting the notion that targeting LINC00473 is an effective strategy to specifically block NSCLC growth. Finally, we identified a function for LINC00473 in regulating gene expression. Collectively, this study reveals mechanistic insights into the roles of LINC00473 in lung cancers and supports the use of LINC00473 as a biomarker and a therapeutic target for LKB1-inactivated lung cancers.

\section{Methods}

Additional experimental details are included in Supplemental Methods.

lncRNA microarray analysis. Total RNAs were extracted from A549 cells after the transduction of WT LKB1, LKB1 K78I mutant, or control retroviruses for 96 hours, and two biological replicates were set up. RNAs were subjected to human lncRNA expression microarray (V3.0) analyses (ArrayStar). The microarray data were deposited in NCBI Gene Expression Omnibus (GEO GSE73414). Genes with an absolute fold change of $\geq 2$ and $P$ value $<$ 0.05 were considered as significantly differentially expressed.

NanoString nCounter gene expression analysis. NanoString gene expression assay analyses were performed according to the manufacturer's protocols (NanoString Technologies) using our customized nCounter GX CodeSet. In brief, total RNA from cultured cells (100 ng) or RNA from FFPE tissues (150-200 ng) were hybridized with the specific capture probes and barcoded reporter probes at $65^{\circ} \mathrm{C}$ for 18 hours and then loaded into the nCounter Pre-station for purification of the hybridized probes. Data collection was performed on the nCounter Digital Analyzer, which counted and tabulated the individual fluorescent barcodes for target RNA molecules in each sample following the manufacturer's instructions. The raw data were analyzed with nSolver Analysis Software for gene expression analysis. The heatmap was generated with the Heatmap.2 R package (https://cran.r-project.org/web/packages/gplots/ index.html) using the normalized NanoString expression data.

RNA ISH. RNA ISH was performed on FFPE xenograft tumors and tissue microarrays (TMAs) using an RNAscope 2.0 HD Reagent Kit (BROWN 310033 or RED 310034, Advanced Cell Diagnostics [ACD]). Briefly, tissue sections were deparaffinized with xylene and $100 \%$ ethanol, and then incubated with pretreat 1 solution for 10 minutes, pretreat 2 for 15 minutes, and pretreat 3 for 30 minutes (Pretreatment kit 310020, ACD). The slides were then hybridized with a custom probe, Hs-LINC00473 tv1 (targeting 781-1755 of NR_026860.1), in the HybEZ oven (ACD) at $40^{\circ} \mathrm{C}$ for 2 hours. The Hs-PPIB probe for human housekeeping gene PPIB was used as a control to ensure RNA quality. After hybridizations, slides were subjected to signal amplification using an HD 2.0 Detection Kit, and hybridization signal was detected using a mixture of solutions A and B (1:60). After counterstaining with hematoxylin, slides were dried in a $60^{\circ} \mathrm{C}$ dry oven for 15 minutes and mounted with EcoMount (BioCare Medical, EM897L). The stained sections were scanned and digitized with Aperio Imagescope (Leica).

RNA-FISH. RNA-FISH was performed using LINC00473 Stellaris FISH probes labeled with Quasar 570 (LGC Biosearch Technologies) following the manufacturer's protocol. Imaging was performed immediately using a Leica DM6000B fluorescence microscope.

Proteomic analysis of the LINCOO473-associated protein complex. lncRNA was first transcribed in vitro using the MEGAscript T7 Transcription Kit (AM1333, Life Technologies) according to the manufacturer's instructions. Identification of lncRNA-interacting protein complexes was performed as previously described, with modifica- 
tions $(54,55)$. Briefly, RNAs were covalently linked to adipic acid dihydrazide agarose beads by periodate oxidation of the RNA 3'-OH terminus. The beads bound with RNAs were incubated with nuclear extracts of A549 cells to pull down the lncRNA-interacting proteins. After extensive washing, beads were boiled with a loading buffer to elute the lncRNA-interacting proteins, which were further separated by SDS-PAGE and subjected to mass spectrometric analysis.

Mouse xenograft studies. Luciferase-expressing A549 cells or H157 cells infected with control shRNA or shLINC00473 lentiviruses were subcutaneously injected to NOD/SCID mice (The Jackson Laboratory). Tumors were measured, and bioluminescence imaging was performed as previously described (56).

Analysis of RNA sequencing and clinical data. RNA sequencing data from the LUAD dataset of the TCGA sequencing project and the accompanying clinical data (40) were used for analysis. Normalized read counts (RSEM; http://deweylab.github.io/RSEM/) for each LUAD sample aligned to LINC00473 were obtained and used for the survival curve analysis. LUAD samples with $\geq 90$ th percentile of LINC00473 expression and those with <90th percentile were considered as high and low groups, respectively. The overall survival was analyzed by Kaplan-Meier curves and log-rank test for all LUAD patients. $P$ values less than 0.05 were considered statistically significant.

Statistics. Data from qRT-PCR, reporter assay, cell proliferation, apoptosis, and in vivo xenograft experiments were analyzed using 2-tailed Student's $t$ test. Results are expressed as mean and \pm SD, and a $P$ value less than 0.05 was considered statistically significant. The biological replicates for each experiment are indicated in the figure legends.

Study approval. All mouse experiments were performed in accordance with a protocol approved by the IACUC of the University of Florida. This study has qualified as exempt review for human subjects research by the University of Florida Institutional Review Board.

\section{Author contributions}

ZC, JLL, and SL collected data, analyzed, and interpreted data and wrote the manuscript. CC, RY, and DAF collected data. MBC, NTG, $\mathrm{EBH}, \mathrm{MBS}$, and WDC provided study materials, analyzed data, and wrote the manuscript. JL, ALA, and FJK analyzed and interpreted data and wrote the manuscript. LW conceived and designed the study, analyzed and interpreted data, and wrote the manuscript.

\section{Acknowledgments}

We thank Ekaterina V. Gavrilova for technical assistance with the RNA FISH experiments. L. Wu is supported by the NIH National Cancer Institute (NCI; R21CA187730), the NIH National Institute of Dental and Craniofacial Research (NIDCR; R01DE023641), and a UF Health Cancer Center Grant. S. Lin is a Damon RunyonSohn Pediatric Fellow supported by the Damon Runyon Cancer Research Foundation (DRSG-7-13). W.D. Cress, M.B. Schabath, and E.B. Haura are supported by a Specialized Programs of Research Excellence (SPORE) grant (P50 CA119997) and by the Tissue Core Facility at the H. Lee Moffitt Cancer Center and Research Institute, an NCI-designated Comprehensive Cancer Center (P30-CA076292). M.B. Carper is supported by an NIH/ NIDCR T90 training grant (T90-DE021986). A.L. Amelio is supported by a Howard Temin Pathway to Independence Award in Cancer Research from the NCI (R00-CA157954).

Address correspondence to: Lizi Wu, UF Health Cancer Center University of Florida, 2033 Mowry Road, Gainesville, Florida 32610-3633, USA. Phone: 352.273.8205; E-mail:lzwu@ufl.edu.

Shuibin Lin's present address is: The Stem Cell Program at Boston Children's Hospital, Department of Biological Chemistry and Molecular Pharmacology, Harvard Medical School, Boston, Massachusetts, USA.
1. Jemal A, Bray F, Center MM, Ferlay J, Ward E, Forman D. Global cancer statistics. CA Cancer J Clin. 2011;61(2):69-90.

2. Janku F, Stewart DJ, Kurzrock R. Targeted therapy in non-small-cell lung cancer - is it becoming a reality? Nat Rev Clin Oncol. 2010;7(7):401-414.

3. Koivunen JP, et al. EML4-ALK fusion gene and efficacy of an ALK kinase inhibitor in lung cancer. Clin Cancer Res. 2008;14(13):4275-4283.

4. Paez JG, et al. EGFR mutations in lung cancer: correlation with clinical response to gefitinib therapy. Science. 2004;304(5676):1497-1500.

5. Shackelford DB, Shaw RJ. The LKB1-AMPK pathway: metabolism and growth control in tumour suppression. Nat Rev Cancer. 2009;9(8):563-575.

6. Alessi DR, Sakamoto K, Bayascas JR. LKB1dependent signaling pathways. Annu Rev Biochem. 2006;75:137-163.

7. Hemminki A, et al. A serine/threonine kinase gene defective in Peutz-Jeghers syndrome. Nature. 1998;391(6663):184-187.

8. Giardiello FM, et al. Increased risk of cancer in the Peutz-Jeghers syndrome. N Engl JMed. 1987;316(24):1511-1514.

9. Beroukhim R, et al. The landscape of somatic copy-number alteration across human cancers.
Nature. 2010;463(7283):899-905.

10. Ding L, et al. Somatic mutations affect key pathways in lung adenocarcinoma. Nature. 2008;455(7216):1069-1075.

11. Matsumoto S, et al. Prevalence and specificity of LKB1 genetic alterations in lung cancers. Oncogene. 2007;26(40):5911-5918.

12. Sanchez-Cespedes M, et al. Inactivation of LKB1/ STK11 is a common event in adenocarcinomas of the lung. Cancer Res. 2002;62(13):3659-3662.

13. Ji H, et al. LKB1 modulates lung cancer differentiation and metastasis. Nature. 2007;448(7155):807-810.

14. Cao C, et al. Role of LKB1-CRTC1 on glycosylated COX-2 and response to COX-2 inhibition in lung cancer. J Natl Cancer Inst. 2015;107(1):358.

15. Shackelford DB, et al. LKB1 inactivation dictates therapeutic response of non-small cell lung cancer to the metabolism drug phenformin. Cancer Cell. 2013;23(2):143-158.

16. Chen Z, et al. A murine lung cancer co-clinical trial identifies genetic modifiers of therapeutic response. Nature. 2012;483(7391):613-617.

17. Carretero J, et al. Integrative genomic and proteomic analyses identify targets for Lkb1deficient metastatic lung tumors. Cancer Cell.
2010;17(6):547-559.

18. Koyama S, et al. STK11/LKB1 deficiency promotes neutrophil recruitment proinflammatory cytokine production to suppress $\mathrm{T}$ cell activity in the lung tumor microenvironment. Cancer Res. 2016;76(5):999-1008.

19. Schabath MB, et al. Differential association of STK11 TP53 with KRAS mutation-associated gene expression, proliferation immune surveillance in lung adenocarcinoma [published online ahead of print October 19, 2015]. Oncogene. doi:10.1038/onc.2015.375.

20. Zheng B, et al. Oncogenic B-RAF negatively regulates the tumor suppressor LKB1 to promote melanoma cell proliferation. Mol Cell. 2009;33(2):237-247.

21. Boudeau J, et al. MO25alpha/beta interact with STRADalpha/beta enhancing their ability to bind, activate and localize LKB1 in the cytoplasm. EMBO J. 2003;22(19):5102-5114.

22. Esteller M, et al. Epigenetic inactivation of LKB1 in primary tumors associated with the Peutz-Jeghers syndrome. Oncogene. 2000;19(1):164-168.

23. Rinn JL, Chang HY. Genome regulation by long noncoding RNAs. Annu Rev Biochem. 
2012;81:145-166.

24. Iyer MK, et al. The landscape of long noncoding RNAs in the human transcriptome. Nat Genet. 2015;47(3):199-208.

25. Ulitsky I, Bartel DP. lincRNAs: genomics, evolution, and mechanisms. Cell. 2013;154(1):26-46.

26. Djebali S, et al. Landscape of transcription in human cells. Nature. 2012;489(7414):101-108.

27. Cabanski CR, et al. Pan-cancer transcriptome analysis reveals long noncoding RNAs with conserved function. RNA Biol. 2015;12(6):628-642.

28. Du Z, et al. Integrative genomic analyses reveal clinically relevant long noncoding RNAs in human cancer. Nat Struct Mol Biol. 2013;20(7):908-913.

29. Gibb EA, et al. Human cancer long noncoding RNA transcriptomes. PLoS One. 2011;6(10):e25915.

30. Hacisuleyman E, et al. Topological organization of multichromosomal regions by the long intergenic noncoding RNA Firre. Nat Struct Mol Biol. 2014;21(2):198-206.

31. Gutschner T, et al. The noncoding RNA MALAT1 is a critical regulator of the metastasis phenotype of lung cancer cells. Cancer Res. 2013;73(3):1180-1189.

32. Gupta RA, et al. Long non-coding RNA HOTAIR reprograms chromatin state to promote cancer metastasis. Nature. 2010;464(7291):1071-1076.

33. Vaahtomeri K, Makela TP. Molecular mechanisms of tumor suppression by LKB1. FEBS Lett. 2011;585(7):944-951.

34. Geiss GK, et al. Direct multiplexed measurement of gene expression with color-coded probe pairs. Nat Biotechnol. 2008;26(3):317-325.

35. Kaufman JM, et al. LKB1 loss induces characteristic patterns of gene expression in human tumors associated with NRF2 activation and attenuation of PI3K-AKT. J Thorac Oncol. 2014;9(6):794-804.

36. Goodwin JM, Svensson RU, Lou HJ, Winslow
MM, Turk BE, Shaw RJ. An AMPK-independent signaling pathway downstream of the LKB1 tumor suppressor controls Snail1 and metastatic potential. Mol Cell. 2014;55(3):436-450.

37. Barretina J, et al. The Cancer Cell Line Encyclopedia enables predictive modelling of anticancer drug sensitivity. Nature. 2012;483(7391):603-607.

38. Tonon G, et al. t(11;19) (q21;p13) translocation in mucoepidermoid carcinoma creates a novel fusion product that disrupts a Notch signaling pathway. Nat Genet. 2003;33(2):208-213.

39. Adzhubei IA, et al. A method and server for predicting damaging missense mutations. Nat Methods. 2010;7(4):248-249.

40. Cancer Genome Atlas Research Network. Comprehensive molecular profiling of lung adenocarcinoma. Nature. 2014;511(7511):543-550.

41. Gu Y, et al. Altered LKB1/CREB-regulated transcription co-activator (CRTC) signaling axis promotes esophageal cancer cell migration and invasion. Oncogene. 2012;31(4):469-479.

42. Komiya T, et al. Enhanced activity of the CREB co-activator Crtc1 in LKB1 null lung cancer. Oncogene. 2009;29(11):1672-1680.

43. Reitmair A, Sachs G, Im WB, Wheeler L. C6orf176: a novel possible regulator of cAMPmediated gene expression. Physiol Genomics. 2012;44(2):152-161.

44. Ahn S, Olive M, Aggarwal S, Krylov D, Ginty DD, Vinson C. A dominant-negative inhibitor of CREB reveals that it is a general mediator of stimulusdependent transcription of c-fos. Mol Cell Biol. 1998;18(2):967-977.

45. Wu L, et al. Transforming activity of MECT1-MAML2 fusion oncoprotein is mediated by constitutive CREB activation. EMBO J. 2005; 24(13):2391-2402

46. Geisler S, Coller J. RNA in unexpected places: long non-coding RNA functions in diverse cellular contexts. Nat Rev Mol Cell Biol. 2013;14(11):699-712.

47. Amelio AL, et al. A coactivator trap identifies NONO (p54nrb) as a component of the cAMP-signaling pathway. Proc Natl Acad Sci U S A. 2007;104(51):20314-20319.

48. Feng $Y$, et al. The CRTC1-NEDD9 signaling axis mediates lung cancer progression caused by LKB1 loss. Cancer Res. 2012;72(24):6502-6511.

49. Wapinski O, Chang HY. Long noncoding RNAs and human disease. Trends Cell Biol. 2011;21(6):354-361.

50. Sanchez-Cespedes M. The role of LKB1 in lung cancer. Fam Cancer. 2011;10(3):447-453.

51. Calles A, et al. Immunohistochemical loss of LKB1 is a biomarker for more aggressive biology in KRAS mutant lung adenocarcinoma. Clin Cancer Res. 2015;21(12):2851-2860.

52. Derrien T, et al. The GENCODE v7 catalog of human long noncoding RNAs: analysis of their gene structure, evolution, and expression. Genome Res. 2012;22(9):1775-1789.

53. Tabernero J, et al. First-in-humans trial of an RNA interference therapeutic targeting VEGF and KSP in cancer patients with liver involvement. Cancer Discov. 2013;3(4):406-417.

54. Caputi M, Mayeda A, Krainer AR, Zahler AM. hnRNP A/B proteins are required for inhibition of HIV-1 pre-mRNA splicing. EMBO J. 1999;18(14):4060-4067.

55. Lin S, et al. Proteomic and functional analyses reveal the role of chromatin reader SFMBT1 in regulating epigenetic silencing and the myogenic gene program. J Biol Chem. 2013;288(9):6238-6247.

56. Chen Z, et al. Aberrantly activated AREGEGFR signaling is required for the growth and survival of CRTC1-MAML2 fusion-positive mucoepidermoid carcinoma cells. Oncogene. 2014;33(29):3869-3877. 